\title{
Quality of life in patients with primary biliary cirhrosis and sclerosing cholangitis by SF-36 health survey: A literature of review
}

\author{
Derizal DERIZAL ${ }^{1}$, Ade Kartikasari SEBBA ${ }^{2}$ \\ 'Department of Economics, State Islamic Institute of Bukittinggi, Indonesia \\ ${ }^{2}$ Department of Public Health, Universitas Gadjah Mada, Indonesia
}

Introduction: Primary biliary cirhrosis (PBC) and primary sclerosing cholangitis (PSC) are part of the family of cholestatic liver disease. Therapies or transplantation are using increase and make improvement of patient survival. However, healing or control of the disease is often not accompanied by a full recovery of quality of life (QoL). This study aimed to reviews analyze various of the QoL of patients PBC and PSC.

Methods: This study used journal which published from 2001 to 2020. Ten articles were selected based on the articles using the Short Form Health Survey 36 (SF-36) in measuring the QoL of patients. The collection of mean score for result SF-36 indicator in each article was used to see the factors that significantly influence the QoL of patients.

Results: The results showed there were 8 indicators that showed the QoL of patients based on SF-36. The Bodily Pain, Mental health, Role Emotion, and Social have a high value was marked by the mean score of patients' satisfaction of QoL over 70. The mean score of General Health, Physical Health, Role Physical and vitality is marked lower in all which are indicated by the mean score of patient satisfaction between 40 and 65 .

Conclusions: The mean of two summary scores of SF-36 show that Physical Component Summary (PCS) is lower than Mental Component Score (MCS). The doctors and medical personnel must take quick action to improve the QoL in the form of physical recovery. 